\title{
Motion Planning on Steep Terrain for the Tethered Axel Rover
}

\author{
Pablo Abad-Manterola ${ }^{1}$, Issa A. D. Nesnas ${ }^{2}$, and Joel.W. Burdick ${ }^{1}$ \\ California Institute of Technology ${ }^{1}$ and the Jet Propulsion Laboratory ${ }^{2}$
}

\begin{abstract}
This paper considers the motion planning problem that arises when a tethered robot descends and ascends steep obstacle-strewn terrain. This work is motivated by the Axel tethered robotic rover designed to provide access to extreme extra-planetary terrains. Motion planning for this type of rover is very different from traditional planning problems because the tether geometry under high loading must be considered during the planning process. Furthermore, only round-trip paths that avoid tether entanglement are viable solutions to the problem. We present an algorithm for tethered robot motion planning on steep terrain that reduces the likelihood that the tether will become entangled during descent and ascent of steep slopes. The algorithm builds upon the notion of the shortest homotopic tether path and its associated sleeve. We provide a simple example for purposes of illustration.
\end{abstract}

\section{MOTIVATION AND BACKGROUND}

$\mathrm{S}_{\mathrm{N}}$ OME of the richest potential science targets for future Mars exploration missions lie in terrains that are inaccessible to state-of-the-art Martian rovers, limiting our ability to carry out in situ analysis of these rich opportunities. Example targets include geologic flows on crater walls, ice deposits in the depths of dark craters, bedrock layers in vertical promontories, and extreme terrain underlying the source of methane plumes (see [1,2] for details). Titan, Europa, Enceladus, and the Earth's moon also offer challenging terrains with associated scientific targets. A new generation of exploration robots is needed to access these extreme terrains in order to probe, sample, and measure. New inquiries of this sort could yield significant scientific rewards.

In order to provide access and in situ sampling in extreme terrains, the Jet Propulsion Laboratory (JPL) and the California Institute of Technology have collaborated to develop the Axel rover [1,2]. The minimalistic Axel robot consists of two wheels connected by a central cylindrical body, a caster arm, and an actively controlled tether passing through the caster arm (Fig. 1). The caster arm, in addition to controlling the tether, provides a reaction force against the terrain needed to generate forward motion when travelling on flat ground.

The work of this collaboration between Caltech and the Jet Propulsion Laboratory (JPL) was performed at JPL under contract to the National Aeronautics and Space Administration. The authors sincerely appreciate the sponsorship of the Exploration Systems Mission Directorate and Solar Systems Exploration program: Dr. C. Moore, Dr. S. Khanna, Dr. T.Y. Yan, Dr. J. Cutts, and Dr. K. Reh. We also appreciate the support of Dr. S. Hayati, Dr. R. Volpe and Dr. G. Udomkesmalee of JPL.

Pablo Abad-Manterola and Joel Burdick are with the California Institute of Technology, Pasadena, CA 91125 USA (e-mail: pablo@caltech.edu).

Issa A.D. Nesnas is with the Jet Propulsion Laboratory, Pasadena, CA 91109 USA (phone: 626-318-9002; e-mail: nesnas@jpl.nasa.gov).

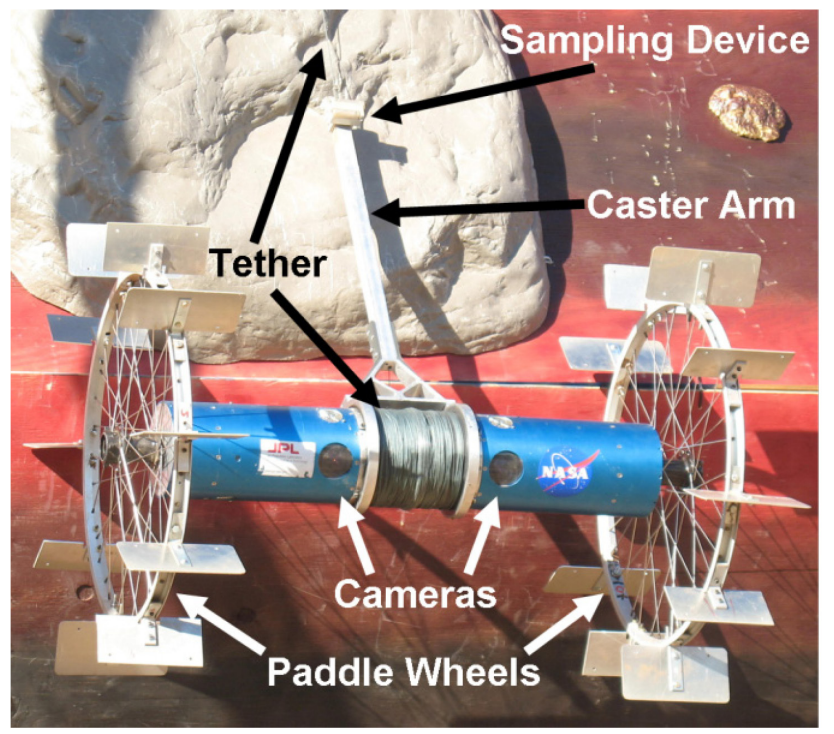

Fig. 1. Photograph of Axel 1 rover with key features labeled.

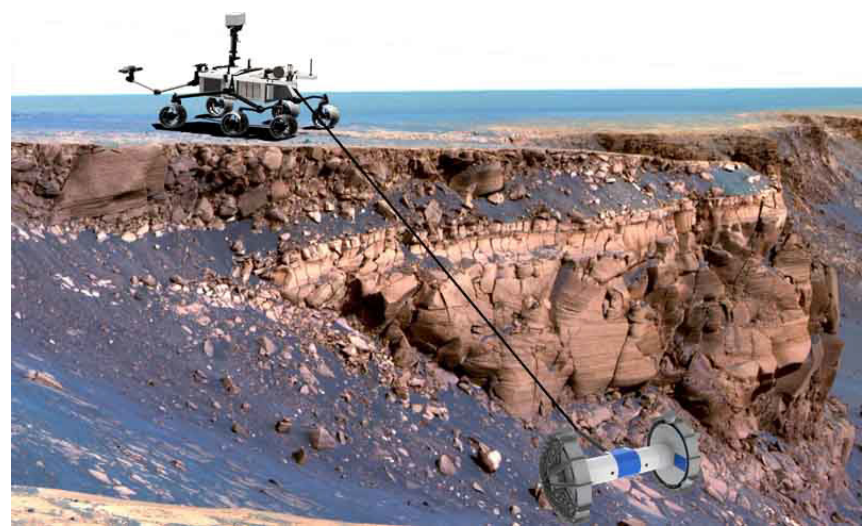

Fig. 2. Proposed mission concept overlaid on false color image of Victoria Crater. Note that the rover graphics are not drawn to scale.

Axel is a rappelling robot which can operate in extreme terrain via the use of a host anchor platform. Since Axel's body acts as a winch (the tether is wound and unwound around the central body as it rotates relative to the wheels), the host platform requirements are reduced to a simple mount. Fig. 2 portrays a hypothetical scenario in which Axel is deployed from the Mars Science Laboratory [3]. Axel has demonstrated high mobility in a simulated Martian environment using both its actively managed tether and its novel grouser (paddle) wheels. The rover has the capability to ascend/descend vertical cliff walls and climb over obstacles whose heights are $\sim 70 \%$ of its wheel diameter $[1,2]$. At times, however, Axel relies on large tether tensions in order to climb and maneuver [1]. An open question is how to autonomously plan Axel's ascending and descending motions 
so as to minimize the likelihood that its tether becomes entangled during long traverses on complex terrains. Additionally, we would like to know how to predict uncontrollable configurations of the rover on steep terrain and avoid them through intelligent planning.

The goal of this paper is to develop the conceptual framework for motion planning algorithms for Axel-like robots operating in steep terrains. While we make many simplifying assumptions to produce this framework, we believe that future work can relax many of these assumptions while staying within the context of the algorithm. After reviewing previous work in the next section, Section III summarizes our simplified model of extreme extra-planetary terrain. Section IV intuitively discusses the steep slope ascent/descent problem and then sketches the planning algorithm. Section V provides technical details behind the algorithm's key steps. Section VI provides an example while Section VII describes open problems and our current activities.

\section{RELATED WORK}

Several prior works have considered the problem of motion planning for tethered robots, e.g. [4-8]. However, in these prior works, the tether acted primarily as an umbilical cord to provide power, communication, and control signals to the mobile robot. The tether did not generate large reaction forces needed for mobility. The primary motion goal in these efforts was to minimize entanglement of the trailing umbilical tether with obstacles [4,5] or with other robots [68 ] in a multi-robot scenario. The motion planning problem considered in this paper is, to our knowledge, unique in that not only must tether entanglement be avoided, but the tether and wheels acting together must also be able to generate sufficient forces in order to ascend or descend steep slopes. Moreover, these forces must be properly aligned with the robot's motion goals to ensure stable robot movement.

Axel is not the first tethered robot to be developed for steep terrain access. The Dante II robot [9-12], designed for descent into an Alaskan volcano, is the most well known tethered robot. The formidable engineering efforts $[9,11]$ behind the development of this vehicle did not produce a detailed theory for tethered robot motion planning, although this work did analyze the forces experienced by a tethered robot on a slope [10]. It is worth pointing out that Dante II's mission ended when large lateral tether forces destabilized and toppled the vehicle during slope ascent. This event demonstrates the need for a planning paradigm that integrates motion planning with tether mechanics and terramechanics.

The JPL Cliffbot system $[13,14]$ is another extra-planetary rover prototype designed to rappel across a cliff face under the actuation of two robotic tethers. While the Cliffbot has traversed cliffs on Svalbard, no motion planning theory for long descents has emerged from that effort, in part because its dual tether design is not suitable for long descents. The analysis presented in this paper assumes a single tether, though many of these principles could be extended to a planning algorithm for Cliffbot-like robots.

It is important to note that Axel's tether is paid out or reeled in from the robot and not from a winch located at the anchor. This approach minimizes abrasion on the tether as compared to a configuration where tether winching is performed at the anchor. While we believe that much of our planning framework can be applied to anchor-winching systems, our focus is on robot-side winching systems.

\section{MODELING EXTREME EXTRA-PlANETARY TERRAIN}

To produce a robust tethered motion planning algorithm, we must first develop a generic blueprint for the extreme extra-planetary terrains that we expect to encounter. Since reaching lunar orbit in June of 2009, NASA's Lunar Reconnaissance Orbiter (LRO) has been acquiring altimetry data to produce a 3-dimensional map of the moon's surface [17]. Fig. 3 shows a portion of this data from the north wall of the Shackleton Crater taken by the LRO's Lunar Orbiter Laser Altimeter (LOLA) [18]. The Shackelton Crater is a good example of an extreme extra-planetary terrain which conventional, un-tethered rovers would be unable to explore, and it is a candidate application for Axel.

\section{Side View of $1 \mathrm{~km}$ Wide Strip of Shackleton Crater}

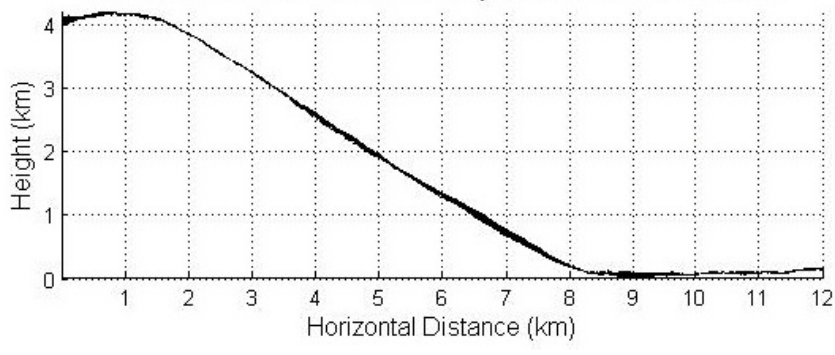

Fig. 3. Elevation map of $1 \mathrm{~km}$ wide strip of the Shackleton Crater taken by LOLA/LRO.

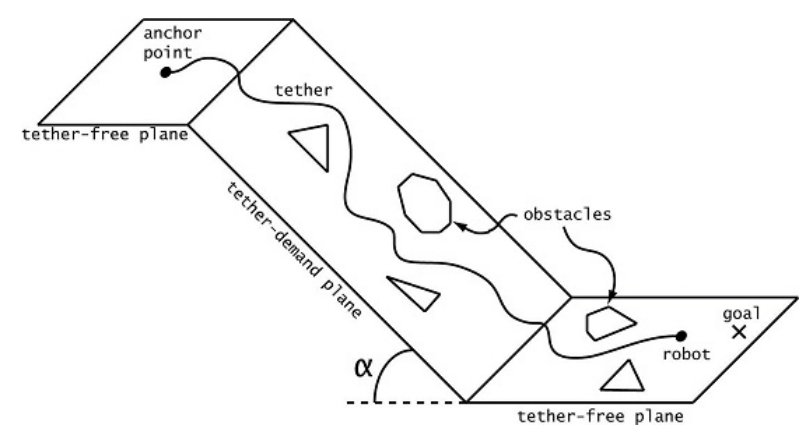

Fig. 4. Simplified 3-plane model of the Shackleton Crater.

The data show that the crater walls have a very consistent and steep slope over $7 \mathrm{~km}$ long. While descending or ascending the slope, a robot must rely upon the tether for climbing or support forces, as beyond a certain slope angle (which depends upon wheel size and geometry, the wheel-soil interaction forces, the robot mass, and the wheels' configuration), the wheels cannot reliably generate sufficient traction forces to propel the robot. Additionally, the crater floor and rim, at least on a macroscopic scale, are relatively flat. Thus, we can conceptualize the rim, crater floor, and slope 
as an intersection of three planes, two which are roughly horizontal and the other sloped at a steep angle $\alpha$ (Fig. 4).

More generally, many extreme terrains of interest can be divided into an alternating sequence of gentle slopes (termed tether-free regions where the rover can travel without the aid of its tether) and steep slopes (termed tether-demand regions because tether forces are essential for mobility in these regions). For example, the promontory in the Victoria crater shown in Fig. 2 can be divided into a reasonably small number of tether-free and tether-demand regions. One can easily conceive of a model consisting of hundreds or thousands of intersecting planes in order to approximate a terrain. And in fact, most available altimetry data for extreme terrains comes in the form of a triangulated mesh. Modeling the crater this way, we change a motion planning problem on a complicated 3-dimensional surface into a quasi-2dimensional problem. For the bulk of this paper we analyze the simple geometry of Figure 4 and sketch in Section VII how to extend the methodology to more realistic surface meshes.

\section{OVERVIEW OF EXTREME TERRAIN Motion PLANNING}

This section considers the conceptual problem of planning the motions of a tethered, wheeled robot on an extreme terrain of the type discussed in Section III in order to motivate the planning approach which is developed in Section V.

\section{A. Summary of the Problem}

We assume that a model of the terrain is a priori known. Any future mission to an extreme extra-planetary terrain will likely incorporate an orbiter which can provide altimetry and high resolution images from which terrain models can be constructed with features on the order of a few meters in scale. We further assume that the terrain is divided into tether-demand and tether-free regions (see Section V). Tetherfree and tether-demand planes may contain one or more convex, polygonal obstacles (non-convex obstacles can be decomposed into a finite number of convex obstacles). We assume that an anchor point, $\mathbf{a}_{0}$, has been chosen. While in practice the anchor may lie well away from a steep slope, we simply assume that $\mathbf{a}_{0}$ is on the edge of a tether-demand plane. The tether is fixed to the anchor, but its geometry is otherwise governed by its interaction with the terrain and with the robot. Likewise, the coordinates of a goal location, g, are given.

The tethered motion planning problem on extreme terrain is formulated in the following manner: given a terrain model consisting of tether-free and tether-demand intersecting planes, a set of obstacles, an initial tethered robot configuration and tether anchor point, compute a feasible roundtrip path from the anchor point to the goal configuration and back. In some cases, more than one feasible path may exist, and thus an optimal path could be chosen based on different criteria, such as "safest" or "shortest." Here, a feasible path is one where, to the resolution of the available terrain model and surface characteristics, Axel is controllable (Section
V.A) at all times during ascent and descent.

\section{B. Ascent/Descent Approach}

Within the tether-free regions, assuming that it is possible to pay out the tether at the same rate that the rover moves, one can use existing motion planning algorithms (e.g., [4]) to compute feasible paths. Motion planning in the tetherdemand planes requires more consideration.

Tethered robot ascent of steep slopes is generally more difficult than descent. Working against gravity, terrains with little or no traction can be very difficult to traverse, and it can be easy for a rover to become stuck underneath an obstacle. Additionally, while executing a tethered ascent, a robot's motion is constrained in that it is unable to deviate much from the path of the tether.

For the reasons just stated, not all feasible descent paths will be feasible ascent paths. Therefore, the set of all possible descent paths will generally be much larger than the set of viable ascent paths. Thus, in order to reduce the computational complexity of the planning problem and to structure the search space, the feasible ascent paths are computed first. We then search for safe descent paths within the set of paths whose initial tether configurations are homotopic (smoothly deformable) to the tether configuration of ascent paths.

\section{Ascent Path Planning}

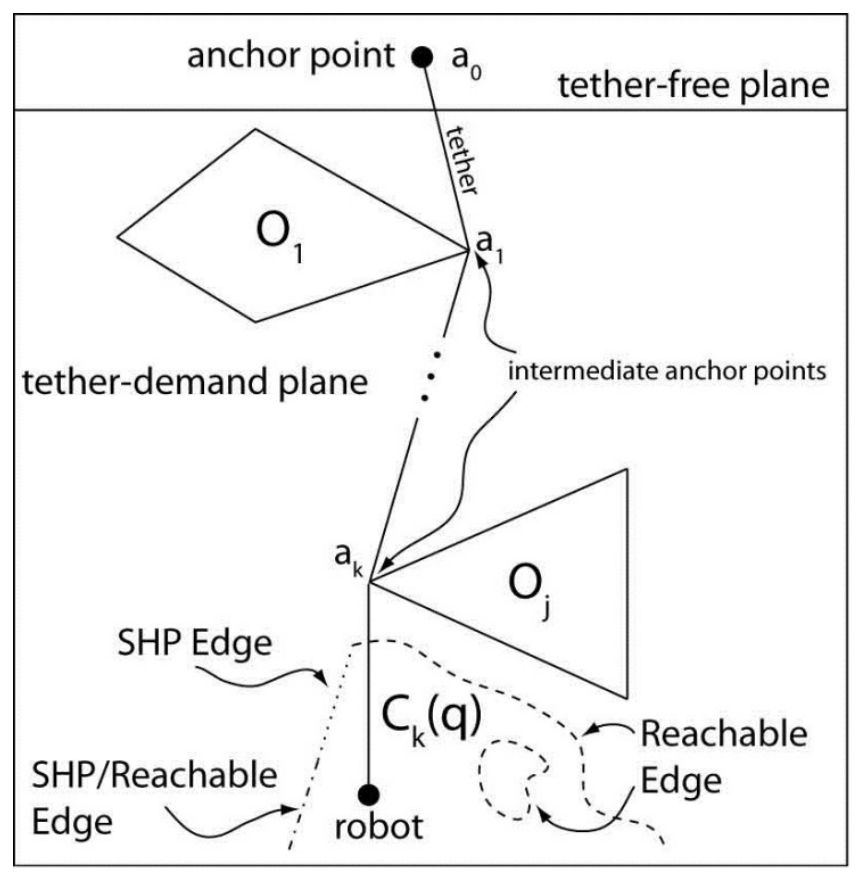

Fig. 5. Top view of intersecting tether-demand and tether-free planes with obstacles and intermediate anchor points. The dotted sections represent the controllable sets.

To climb a tether-demand plane, Axel must reel in its tether until it is taut and use the cable's tension to travel up the steep slope. The shape of the taut tether is very important to the robot's ascent since it will dictate the direction in which this upward force is applied. For simplicity, we will assume that the contact between the tether and the ground is frictionless. This ignores "frictional" obstacles, or snagging points 
that may occur in areas of high surface friction. With this assumption, the taut tether geometry will be the shortest obstacle-free path from the anchor to the robot. Hence, one can compute the configuration of a taut tether given a slack configuration by finding the shortest homotopic path (SHP) from the anchor to the robot's configuration. I.e., this is the shortest path which is smoothly deformable to the tether's geometry. An algorithm for computing the SHP in a 2-D plane with obstacles can be found in [19]. Aspects of this algorithm's construction will be summarized in Section V.

Once the SHP from the anchor to a given robot configuration has been computed, we can identify intermediate anchor points. These are the points at which the taut tether contacts one or more of the obstacles, $\mathrm{O}_{1} \ldots \mathrm{O}_{\mathrm{N}}$. Starting with the anchor point, $\mathbf{a}_{\mathbf{0}}$, the intermediate anchor points, $\mathbf{a}_{\mathbf{1}} \ldots \mathbf{a}_{\mathbf{k}}$, are indexed in increasing order along the tether from the anchor point (Fig. 5). An intermediate anchor point, $\mathbf{a}_{\mathbf{j}}$, is passable from robot configuration $q$ if, given $q$ and an SHP with anchor points $\mathbf{a}_{\mathbf{0}} \ldots \mathbf{a}_{\mathbf{j}}$, the robot can reach a configuration which removes $\mathbf{a}_{\mathbf{j}}$ from the SHP and makes $\mathbf{a}_{\mathbf{j}-1}$ the most immediate anchor point.

With these definitions, the ascent path planning problem simplifies to finding a taut tether configuration containing a sequence of passable anchor points between $\mathbf{g}$ and $\mathbf{a}_{\mathbf{0}}$. To find the set of all feasible ascents, one computes all of the SHPs connecting the anchor, $\mathbf{a}_{\mathbf{0}}$, to the goal, g. Algorithms for finding the shortest path of given homotopy type already exist [19-21]. We only consider shortest paths which do not wind around an obstacle. Let $\left\{\mathbf{S}_{\mathrm{i}}\left(\mathbf{a}_{\mathbf{0}}, \mathbf{g}\right)\right\}, \mathrm{i}=1, . . \mathrm{N}_{\mathbf{s}}$, denote this set of taut tether paths connecting $\mathbf{a}_{\mathbf{0}}$ to $\mathbf{g}$. Assuming a finite number of obstacles in the region of interest, $\mathrm{N}_{\mathbf{s}}$ is finite. Likewise, there are a finite number of intermediate anchor points, $A_{i}$, in path $\mathbf{S}_{\mathrm{i}}\left(\mathbf{a}_{\mathbf{0}}, \mathbf{g}\right)$.

To determine whether or not the intermediate anchors of $\mathbf{S}_{\mathrm{i}}\left(\mathbf{a}_{\mathbf{0}}, \mathbf{g}\right)$ are passable, we define a controllable set, $\mathbf{C}_{\mathbf{i}, \mathbf{j}}(\mathrm{q})$, associated with the $\mathrm{j}^{\text {th }}$ anchor point of the $\mathrm{i}^{\text {th }} \mathrm{SHP}, \mathbf{a}_{\mathbf{i}, \mathbf{j}}$. $\mathbf{C}_{\mathrm{i}, \mathrm{j}}(\mathrm{q})$ is the set of points that, given the SHP associated to $q$ and the closest intermediate anchor $\mathbf{a}_{\mathbf{j}}$, are reachable from the current robot configuration, $q$. Generally, the controllable sets will depend upon the SHP, the terrain angle, the terrain traction model, and the robot's dynamic capabilities.

Controllable sets have 3 types of edges: 1) an edge which, when crossed, changes the list of anchor points in the SHP (SHP edge), 2) an edge which is the limit of reachable configurations (reachable edge), and 3) an edge which may be both (1) and (2). In Fig. 5, the SHP edge is the dotted line, the reachable edges are the dashed lines, and the SHP/Reachable edge is the dotted-dashed line.

From the definitions presented in this section, it should be evident that the intermediate anchor $\mathbf{a}_{\mathbf{i}, \mathbf{j}}$ is passable if $\mathbf{C}_{\mathbf{i}, \mathbf{j}}(\mathrm{q})$ has a SHP edge which, when crossed, makes $\mathbf{a}_{\mathbf{i}, \mathbf{j}-\mathbf{1}}$ the most immediate anchor point. Once an intermediate anchor is found to be passable, the crossed SHP edge is used to calculate a starting configuration, $q$, for the analysis of the following controllable set. Optionally, $q$ becomes set-valued as the starting point for the computation of $\mathbf{C}_{\mathbf{i}, \mathbf{j}-\mathbf{1}}(q)$.

If all intermediate anchor points of $\mathbf{S}_{\mathrm{i}}\left(\mathbf{a}_{\mathbf{0}}, \mathbf{g}\right)$ are passable, a kinodynamic motion planning algorithm [22] can be used to search for feasible or optimal paths from $\mathbf{g}$ to $\mathbf{a}_{\mathbf{0}}$ within the space of the associated controllable sets.

\section{Descent Path Planning}

The descent path planning problem is similar to the ascent planning problem except that we are now further constrained to consider only paths which are homotopic to the feasible ascent paths, $\left\{\mathbf{S}_{\mathrm{i}}^{\text {feas }}\left(\mathbf{a}_{\mathbf{0}}, \mathbf{g}\right)\right\}$. As described below we use the sleeve framework of Hershberger and Snoeyink [19] to search for descent paths within the feasible ascent homotopy classes.

\section{E. Summary}

The basic tethered robot steep terrain planning algorithm can be summarized in the following pseudo-code.

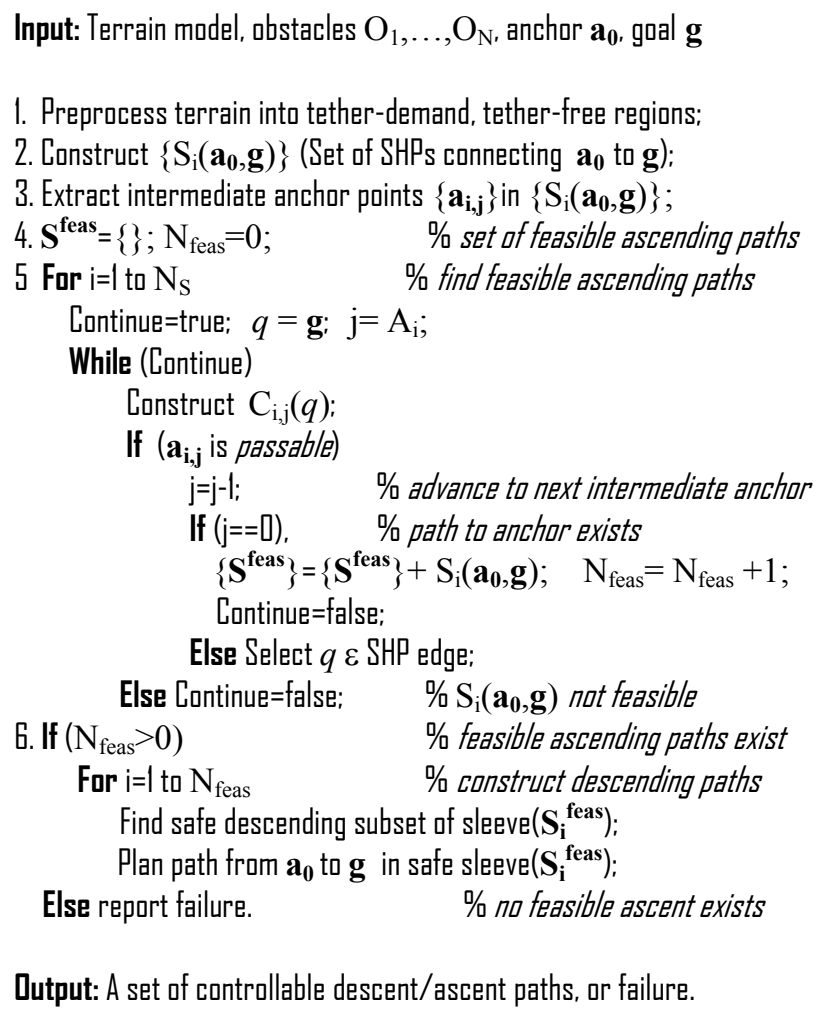

Dutput: A set of controllable descent/ascent paths, or failure.

Fig. 6. Pseudo-code of steep terrain tethered robot planning algorithm

\section{Algorithm DETAILS}

This section provides additional technical details regarding the key steps of the planning algorithm described above.

\section{A. Homotopies (Sleeves) of Ascending Paths}

To construct the taut tether configuration and to preprocess the terrain for efficient descent planning, the tether demand regions are triangulated as follows. Recall that a 2dimensional simplicial complex is a triangulated 2-manifold. I.e., it is a collection of triangles, edges, and vertices such that individual triangles may have only 3 relations 1) no intersection, 2) intersection at a vertex, or 3) intersection at two vertices and a common edge. A boundary-triangulated 2-manifold (BTM) is a simplicial complex in which all ver- 
tices are incident to two boundary edges. Boundary edges are incident only to a single triangle. Practically, boundary edges form the boundaries of the tether demand regions or the edges bounding obstacles. Fig. 7 shows the tetherdemand plane of Fig. 5 triangulated as a BTM. [19] provides details on constructing a BTM.

A simple path is a curve which does not cross itself. We are concerned only with simple paths since we are modeling a continuous tether and do not want paths that form closed loops around obstacles.

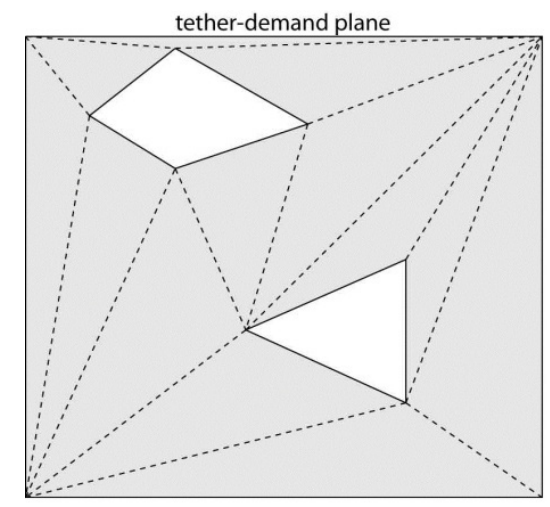

Fig. 7. The tether-demand plane of Fig. 5 processed into a boundarytriangulated 2-manifold (BTM).

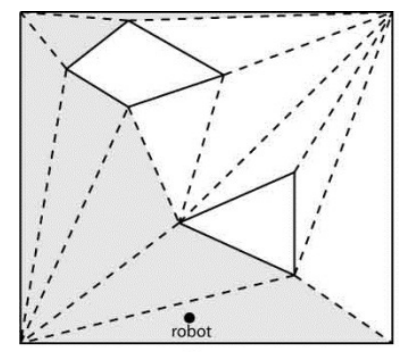

a.

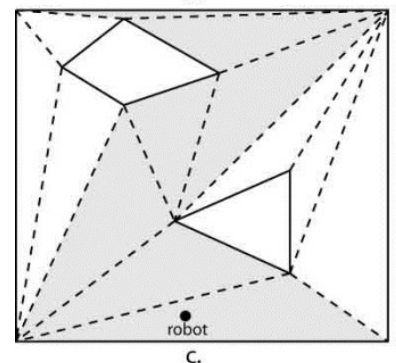

Fig. 8(a)-(d). Shaded grey regions represent the four possible sleeves running from the top of the tether-demand plane to the robot configuration.

Finally, a sleeve [19] is a triangulated simple polygon (a polygon with no holes) whose dual tree is a simple path. Sleeves can represent the homotopy class of the tether configuration during ascent. For example, Fig. 8 shows the four possible sleeves for the BTM presented in Fig. 7. These sleeves represent the 4 unique homotopy classes of paths connecting the top of the tether demand region to the robot configuration. The sleeve is particularly useful because it has the property that any two simple paths in the same sleeve with the same endpoints must be homotopic. Thus, all tether paths within the sleeve of $\mathbf{S}_{\mathrm{i}}\left(\mathbf{a}_{0}, \mathbf{g}\right)$, denoted by sleeve $\left[\mathbf{S}_{\mathbf{i}}\left(\mathbf{a}_{\mathbf{0}}, \mathbf{g}\right)\right]$, will deform to $\mathbf{S}_{\mathbf{i}}\left(\mathbf{a}_{\mathbf{0}}, \mathbf{g}\right)$ when the tether is pulled taut. The shortest path in a simple polygon can be readily found. While it is not relevant to this paper, it is worth noting that given a slack tether shape, the associated SHP (taut tether configuration) can be found from the sleeve construction [19].

Let $\mathbf{S}_{\mathrm{i}}^{\text {feas }}\left(\mathbf{a}_{0}, \mathbf{g}\right)$ denote the $\mathrm{i}^{\text {th }}$ feasible ascending SHPs (all of its intermediate anchors are passable). After all feasible ascent paths, $\left\{\mathbf{S}_{\mathrm{j}}^{\text {feas }}\left(\mathbf{a}_{0}, \mathbf{g}\right)\right\}, \mathrm{j}=1, \ldots, \mathrm{N}_{\text {feas }}$ are found, the search space for descending paths is limited to the sleeves of the feasible ascent paths, e.g., one of the shaded regions of Fig. 8. As described below, we can further refine this space by looking only at the subsets of these regions where the rover is safe and controllable. If no suitable descent path can be found within this space, we can expand the search to include triangles adjacent to the sleeve, so long as the path returns to the sleeve through the same triangle edge from which it left. This will ensure homotopy to the ascent configuration when the tether is pulled taut.

\section{B. Constructing Controllable Sets (Safe Rover Postures)}

During the ascent planning, we must construct the controllable sets needed to assess if an intermediate anchor is passable. In the descent phase, it is useful to determine the subsets of the sleeves where the vehicle is likewise safe and controllable. In general, constructing reachable sets of nonlinear control systems is a difficult task and depends upon the dynamic capabilities of the robot, its configuration on the terrain, a terrain-vehicle interaction model, and the geometry of the tether. Here we sketch a general and conservative approach.

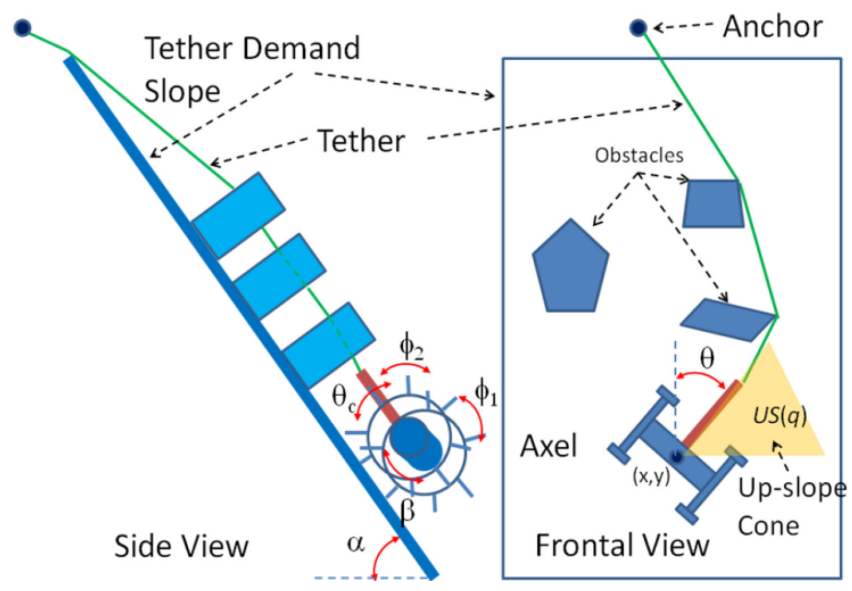

Fig. 9. Sketch of Axel in tether-demand, showing Axel configuration variables and up-slope motion cone, $U S(q)$

Controllability requires a dynamic model which characterizes the vehicle's ability to generate maneuvering forces. Let $L(q, \dot{q})=K(q, \dot{q})-V(q)$ be the rover's Lagrangian, where $K$ is its kinetic energy and $V$ its potential energy, and $q$ denotes its configuration variables: $q=\left(x, y, \theta, \beta, \varphi_{1}, \varphi_{2}, \theta_{c}\right)$ for Axel, where $\mathrm{x}, \mathrm{y}$, and $\theta$ refer to Axel's position on the tether demand plane and its upslope orientation, respectively. The variables $\beta, \varphi_{1}$, and $\varphi_{2}$ denote the body and wheel angles and $\theta_{\mathrm{c}}$ is the caster arm angle.

To obtain the correct equations of motion, we must apply appropriate constraints. The tether constrains the system 
such that the distance between Axel and the upslope intermediate anchor can be no greater than the length of unreeled tether (assuming that tether elongation under load is negligible). The movement of the caster arm is restricted such that it cannot penetrate the ground. Under these assumptions, the tether reaction force and the caster arm ground reaction force can be modeled as a set of two independent holonomic inequality constraints of the form $h_{j}(q) \leq 0, j=1,2$.

A model for the wheel-ground interaction forces can be quite complex and dependent upon the soil type [23]. For purposes of discussion, assume that a Coulomb friction model governs Axel's ground contact. Assuming Axel rolls upon the ground without slipping, its motion is governed by nonholonomic constraints of the form: $\omega_{k}(q) \dot{q}=0$, $k=1,2,3$. Including the reaction forces that arise from the nonholonomic constraints, the Euler-Lagrange equations take the form

$$
\frac{d}{d t}\left(\frac{\partial L}{\partial \dot{q}}\right)-\frac{\partial L}{\partial q}+C^{T}(q) \Lambda=\tau
$$

where $\tau$ is the vector of wheel and caster motor torques, $\Lambda$ are the undetermined Lagrange multipliers that correspond to the tether, caster, and wheel reaction forces (assuming the constraints are active), and matrix $C(q)$ arises from the constraints:

$$
C(q)=\left[\begin{array}{ll}
\omega_{k}^{T}(q) & \left(\frac{\partial h_{j}(q)}{\partial q}\right)^{T}
\end{array}\right]^{T} .
$$

The Euler-Lagrange equation can be arranged in the form

$$
M(q) \ddot{q}+B(q, \dot{q})+G(q)+C^{T}(q) \Lambda=\tau
$$

where $M(q)$ is a mass matrix, $B$ is the Coriolis forces, and $G(q)$ denotes gravitational forces. When active, the holonomic constraints can be modeled as forces acting on the system: $\ddot{z}_{J}=F\left(z_{j}, \dot{z}_{J}\right)$, where $z_{j}=h_{j}(q)$. More generally, this can be expressed as:

$$
\frac{\partial h_{j}}{\partial q} \ddot{q}+\frac{d}{d t}\left[\frac{\partial h_{j}}{\partial q}\right] \dot{q}=F\left(h_{j}(q), \frac{\partial h_{j}}{\partial q} \dot{q}\right) .
$$

The tether tension force, for example, can be readily modeled as a mass-spring-damper system. These forces can be added into the time-derivative of the constraint equation, yielding $C \ddot{q}+\dot{C} \dot{q}+S=0$, where

$$
S=\left[0,0,0,-F\left(z_{j}, \dot{z}_{J}\right)\right]^{T} .
$$

Solving for the Lagrange multipliers,

$$
\Lambda=\left[C M^{-1} C^{T}\right]^{-1}\left[C M^{-1}(\tau-B-G)+S+\dot{C} \dot{q}\right]
$$

substituting the multipliers into Equation (1), and assuming that Axel is moving slowly, yields:

$$
\begin{aligned}
\ddot{q}= & \left(M^{-1}-M^{-1} C^{T}\left[C M^{-1} C^{T}\right]^{-1} C M^{-1}\right)[\tau-G] \\
& -M^{-1} C^{T}\left[C M^{-1} C^{T}\right]^{-1} S \\
\equiv & \left.\left(M^{-1}-K(q) C M^{-1}\right) \tau-G(q)\right]-K(q) S
\end{aligned} .
$$

Since Axel moves slowly, its motions can be approximated by $\dot{q} \approx \ddot{q} \Delta t$ for small time $\Delta t$. Thus, axel can successfully maneuver upslope if there exists at configuration $q$ a feasible set of motor torques $\tau$ such that:

$$
\left.\left(M^{-1}-K(q) C M^{-1}\right) \tau-G(q)\right]-K(q) S \in U S(q)
$$

where $U S(q)$ is the upslope motion cone at configuration $q$, which represents the directions of motions which usefully displace Axel in a direction which allows passing of the nearest intermediate anchor (Fig. 9).

In practice, one or more of Axel's wheels may slip as it maneuvers, and the tether and caster constraints may become intermittently active and inactive. We test if the wheel reaction forces (which are computed from the Lagrange multiplers) do not exceed the friction limits. If they do, the configuration is deemed uncontrollable. Alternatively, one can use a more complex dynamic model which incorporates wheel slip forces. It is generally not possible to model these issues with enough precision to predict when Axel will switch between different models that govern its behavior. Thus, Axel's dynamics represent a multiple model control system [24], and Axel's ability to locally maneuver around an obstacle can be formally posed as a problem in multiple model controllability [24], which is beyond the scope of this paper.

A similar analysis can be used during descent planning to prune the geometry of the sleeve down to the subset of safe and controllable rover configurations. In practice, the controllable sets for ascent and the pruning procedure during descent planning are computed on a grid. Because controllability depends on the SHP, each sleeve corresponding to a feasible ascent path, $\mathbf{S}_{\mathbf{i}}^{\text {feas }}$, is discretized, and the controllability calculation is performed at each point of the grid. Fig. 10 shows a computational example of such a discretized calculation for the sleeve of one ascending SHP.

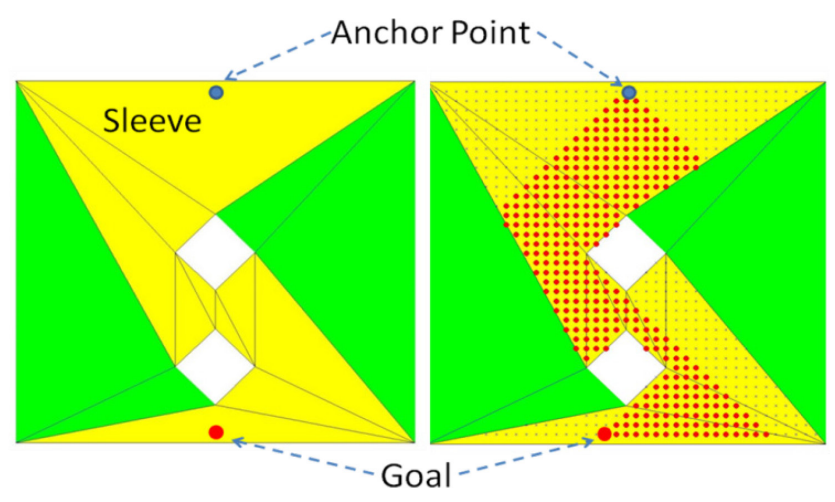

Fig. 10. Computational example of Axel controllability calculation in one sleeve of 45 degree sloped tether demand region. Left figure shows goal and anchor point, with obstacles in white and one possible sleeve in yellow. Dots in right figure represent grid points in sleeve. Red circles depict controllable/safe subset of sleeve.

Finally, the dynamic model, with the tether reaction force removed, can be used to predict if the rover is mobile at a given posture, $q$. This analysis can be used to classify the motion planes in to tether-demand and tether-free categories. 


\section{EXAMPLE}

A simple example will help to illustrate the concepts presented in this paper. Let the terrain model be given as in Fig. 11 , with the anchor point placed near the border between a tether-free and tether-demand plane. Obstacles and a goal are located on the tether-demand plane, which is slanted at angle $\alpha$ to the horizontal. The dotted line denotes the boundary between compact soil and loose sand terrain regions. In this example, the robot is modeled as a simple point mass with Coulomb friction governing its interaction with the terrain.

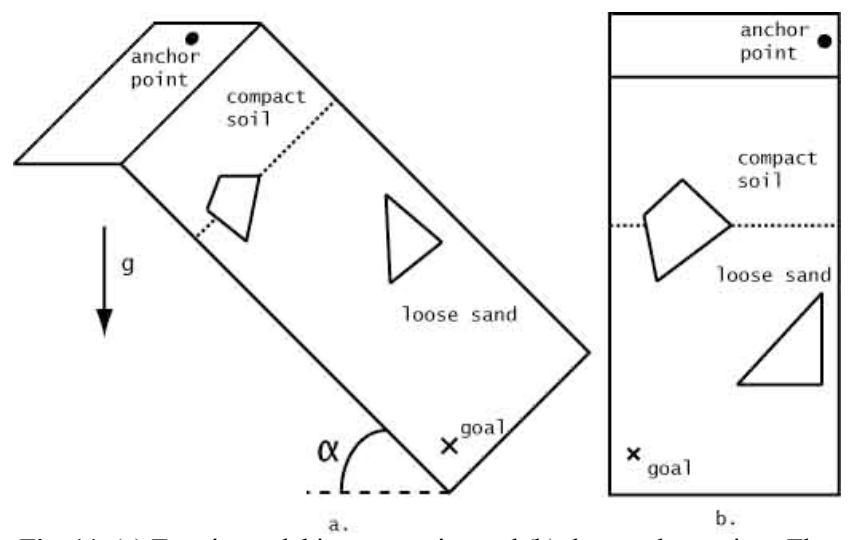

Fig. 11. (a) Terrain model in perspective and (b) the top-down view. The dotted line denotes the boundary between compact soil and the loose sand.

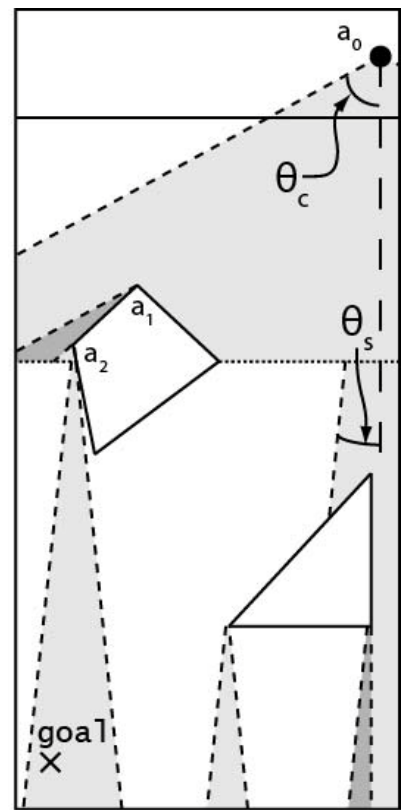

Fig. 12. Shaded regions represent some of the controllable sets for the terrain model. Different shadings distinguish between adjacent controllable sets.

Our first task is to compute the homotopy classes of the viable ascent paths, which requires that we find the controllable sets. If we assume quasi-static robot motion, then the reachable edges of the controllable sets will occur at the points where the point mass robot will start to slip in static equilibrium. A straightforward calculation shows that this relation is given by

$$
\left|\theta_{\max }\right|=\sin ^{-1}(\mu \cot \alpha) .
$$

$\theta_{\max }$ is the maximum angle from the vertical to the straight line connecting the robot to the nearest anchor point, $\alpha$ is the slope angle, and $\mu$ is the coefficient of friction between the robot and the terrain (which differs in the two soil regions). Fig. 12 shows some of the controllable sets in this terrain model. $\theta_{\mathrm{c}}$ is the maximum up-slope tether angle for the compact soil and $\theta_{\mathrm{s}}$ is the analogous value for the loose sand, which has a lower coefficient of friction.

Following the algorithm in Sections IV and V, we see that the only continuous sequence of passable intermediate anchor points from the goal to $\mathbf{a}_{\mathbf{0}}$ is $\left[\mathbf{a}_{\mathbf{1}} \mathbf{a}_{2}\right]$ as shown in Fig. 12. Next, we compute the BTM of the tether-demand plane and search for a path within the ascent sleeve based on an optimization criterion, in this case the shortest path (Fig. 13).

At first glance the naïve shortest path from the anchor point to the goal may seem very desirable because it completely avoids contact between the tether and the obstacles. The controllable sets, however, tell us that this path does not guarantee the robot's safety on this steep terrain. Instead, we take advantage of the passable anchor points in order to find a safe path to the goal and back to the anchor point.

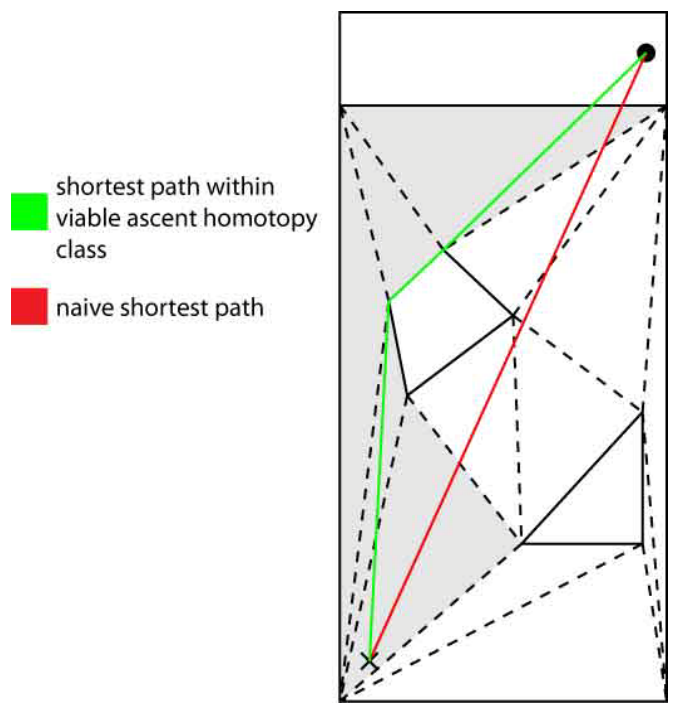

Fig. 13. Dashed lines show the BTM of the terrain, where the shaded region is the sleeve of the viable ascent path,. The red line is a naïve shortest path from anchor to goal, while the green line is the shortest path within the only viable ascent homotopy class.

\section{DISCUSSION AND CONCLUSION}

This paper was motivated by the belief that tethered robots, such as the Axel rover, offer a potential platform to access scientific targets on extreme extra-planetary terrains. This paper presented a first step toward a motion planning framework for such vehicles. Admittedly, our algorithm is based on many assumptions which may not hold in practice. However, we feel that many aspects of this framework will still hold when some of these assumptions are relaxed. We considered only flat tether-demand planes in this paper. However, the BTM construction holds for curved surfaces 
and 3-dimensional surface meshes. Keeping with the assumption of frictionless tether-ground interaction, the shortest path on these non-flat BTMs is equivalent to finding the shortest path for a point robot operating in a field of 3dimensional polyhedral obstacles [25]. The generalization of the notion of sleeve to this geometry is still an open question. A more difficult problem is to include the effects of ground-tether friction. Finally, it is abundantly clear that the motion plans may be quite sensitive to the details of the wheel/soil interaction model. Developing a planner which is robust against errors in this model is essential for real world applications. Finally, an on-line version of a tethered-robot planner which can handle terrains which are only a priori partially or incompletely known will be invaluable in some applications.

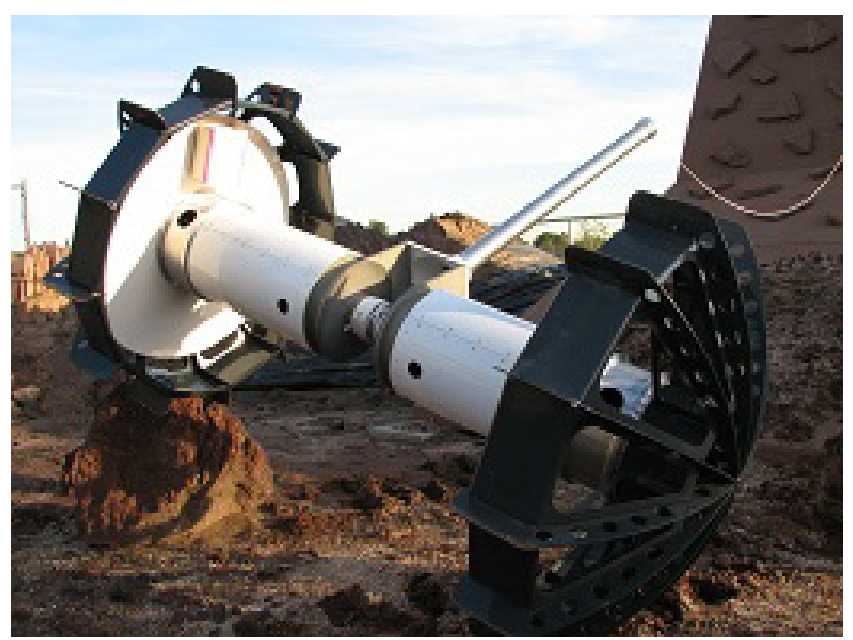

Fig. 14. Assembled Axel II prototype traversing a rock in the JPL Mars Yard.

Our long term plan is to implement this algorithm on the Axel II rover during field experiments conducted on terrains which closely resemble mission targets on the Moon or Mars (see Fig. 14).

Aknowledgements: We would like to thank Dr. J.M. Cameron and Dr. H.D. Nayar of the Jet Propulsion Laboratory for providing the altimeter data from the Shackleton Crater.

\section{REFERENCES}

[1] P. Abad-Manterola, J.A. Edlund, J. W. Burdick, A. Wu, T. Oliver, I. A. D. Nesnas,, and J. Cecava "Axel: A minimalist Tethered Rover for Exploration of Extreme Planetary Terrains," IEEE Robotics and Automation Magazine, vol. 16, no. 4, pp. 44-52, Dec. 2009.

[2] P. Abad-Manterola, J. W. Burdick, I. A. D. Nesnas, "Axel rover paddle wheel design, efficiency, and sinkage on deformable terrain," IEEE Conference on Robotics and Automation, 3-7 May 2010.

[3] http://marsprogram.jpl.nasa.gov/msl/

[4] P. G. Xavier, "Shortest Path Planning for a Tethered Robot or Anchored Cable," Proc. IEEE Int. Conf. Robotics and Automation, pp. 1101-1107, Detroit, May 1999.

[5] S. Hert and V. Lumelsky, "Planar Curve Routing for Tethered-Robot Motion Planning," Int. J. Computational Geometry \& Applications, vo. 8, no.4, pp. 437-466, Aug., 1998.
[6] S. Hert and V. Lumelsky, "Motion Planning in $\mathrm{R}^{3}$ for Multiple Tethered Robots," IEEE Trans. Robotics and Automation, vol. 15, no.4, pp. 623-639, Aug.. 1999.

[7] S. Hert and V. Lumelsky, "The Ties that Bin: Motion Planning for Multiple Tethered Robots," Robotics and Autonomous Systems, vol. 17, no. 3, ppg. 187-215, May 1996.

[8] S. Hert and V. Lumelsky, "Moving multiple tethered robots between arbitrary configurations," Proc. IEEE/RSJ Int. Conf. Intell. Robots and Systems, vol. 2, pp. 280-285, 1995.

[9] J.E. Bares and D.S. Wettergreen, "Dante II: Technical Description and Lessons Learned," Int. J. Robotics Research, vol. 18, no. 7, pp. 621649 , july 1999 .

[10] D. Wettergreen and C. Thorpe, "Developing Planning and Reactive Control for a Hexapod Robot," Proc. IEEE Int. Conf. Robotics and Automation, pp. 2718 2723, Minnesota, April 1996,

[11] M. Krishna, J. Bares, and E. Mutschler, "Tethering System Design for Dante II," Proc. IEEE Int. Conf. Robotics and Automation, pp. 11001105, Albuquerque, NM, April, 1997.

[12] D. Apostolopoulos and J. Bares, "Locomotion Configuration of a Robust Rapelling Robot," Proc. IEEE Int. Conf. Robotics and Automation, vol. 3, pp. 280-284, Aug., 1995.

[13] E. Mumm, S. Farritor, P. Pirjanian, C. Leger, and P. Schenker, "Planetary Cliff Descent Using Cooperative Robots, Autonomous Robots, vol. 16, no. 3, pp.259-272, 2004.

[14] P. Pirjanian, C. Leger, E. Mumm, B. Kennedy, M. Garrett, H. Aghazarian, S. Farritor, and P. Schenker, "Distributed Control for a Modular Reconfigurable Cliff Robot," Proc. IEEE Int. Conf. Robotics and Automation, pp. 4083-4088, Washington D.C., May, 2002.

[15] P. J. McKerrow and D. Ratner, "The Design of a Tethered Aerial Robot," Proc. IEEE Int. Conf. on Robotics and Automation, pp. 255350, Rome, May, 2007

[16] H.-S. Ahn, S.-I. Nah, Y-C. Lee, and W. Yu, "A controller design of a tethered-robot guiding system, Proc. $3^{\text {rd }}$ Int. Conf. on Ubiquitous Robots and Ambient Intelligence, 2006.

[17] http://lunar.gsfc.nasa.gov/

[18] http://lunar.gsfc.nasa.gov/lola.html

[19] J. Hershberger and J. Snoeyink, "Computing Minimum Length Paths of a Given Homotopy Class, Computational Geometry, vol. 4, pp. 6397, 1994.

[20] F. Sciortino, "An Algorithm to Find All Paths between Two Nodes in a Graph," J. Computational Physics, vol. 87, no. 1, pp. 231-236, 1990.

[21] D. Grigoriev and A. Slissenko, "Polytime Algorithm for the Shortest Path in a Homotopy Class amidst Semi-Algebraic Obstacles in the Plane," Preprint.

[22] S. Lavalle, Planning Algorithms, Cambridge Univ. Press, 2006.

[23] H. Shibly, K. Iagnemma, S. Dubowsky, “An Equivalent Soil Mechanics Formulation for Rigid Wheels in Deformable Terrain, with Application to Planetary Exploration Rovers," J. Terramechanics, vol. 42, no. 1, pp. 1-13, Jan. 2005

[24] T. Murphey and J.W. Burdick, "A Local Controllability Test for Nonlinear Multiple Model Systems," Proc. 41 st Conf. on Decision and Control, Dec. 2002.

[25] K. Jiang, L.D. Seneviratne, and S.W.E. Earles, "Three-Dimensional Shortest Path Planning in the Presence of Polyhedral Obstacles," Journal of Mechanical Engineering Science, vol. 210, no. 4, pp. 373-381, 1996. 